\title{
Major depressive disorders in chronic hemodialysis patients in Nazareth: identification and assessment
}

This article was published in the following Dove Press journal:

Neuropsychiatric Disease and Treatment

25 July 2012

Number of times this article has been viewed

\section{Zaher Armaly \\ Joseph Farah \\ Adel Jabbour \\ Bishara Bisharat \\ Amir Abd-El Qader \\ Shahira Saba \\ Maha Zaher \\ Elia El Haj \\ Munir Hamzi \\ Abdalla Bowirrat}

The Nazareth Hospital, Hospital Affiliated with Galilee Medical SchoolBar Ilan University, Zefat, Israel
Correspondence: Abdalla Bowirrat Professor of Neuroscience and Population Genetics, EMMS Nazareth-The Nazareth Hospital, Nazareth, 16100, Israel Tel +97246356133

Fax +97246028882

Email bowirrat@netvision.net.il
Objective: Depression illnesses are commonly observed in hemodialysis (HD) patients, which can influence the quality of life of end-stage renal disease patients. We evaluate the prevalence and predictive risk factors of depression in the Arab population undergoing HD in Nazareth, Israel.

Methods: We conducted a prospective study that included 71 patients in the HD unit with a mean age of $61.9 \pm 14.13$ years who had undergone HD and 26 healthy control subjects with a mean age of $59.3 \pm 7.3$. Beck's Depression Inventory and Hamilton Depression Scale assessments were administered. Blood analysis for hematological and biochemical parameters was obtained. Diagnosis was made using the Diagnostic and Statistical Manual of Mental Disorders scale to correlate psychological variables with clinical, hematological, and biochemical parameters. Statistical analysis was carried out using analysis of variance followed by Tukey post-hoc multiple comparison tests.

Results: The prevalence of depression was $43.7 \%$ in HD patients. Between HD patients and controls, cortisol values were $16.96 \pm 0.5476$ and $11.96 \pm 1.116$, respectively $(P<0.0001$; 95\% confidence intervals [CI]: 2.416-6.825). Between depressed HD patients versus control subjects, cortisol values were $16.48 \pm 0.72$ and $11.96 \pm 1.116$, respectively $(P=0.0013$; 95\% CI: 1.878-7.184). Hematological and biochemical parameters were compared between depressed HD and nondepressed patients, but differences between the two groups were found to be insignificant $(P>0.05)$.

Conclusion: Our HD patients were severely depressed. Studies of glucocorticoid turnover activity such as cortisol, a potent chemical stress hormone, may be used as a model and marker for early diagnosis of depression among HD patients. The strong familial support system in Arabic traditions has failed to decrease depression among these patients.

Keywords: Beck Depression Inventory, cortisol, depression, hemodialysis

\section{Introduction}

Depression is the most common psychological problem encountered in patients with end-stage renal disease (ESRD) ${ }^{1-3}$ and hemodialysis (HD) patients. HD is the most prevalent form of renal replacement therapy, with over $75 \%$ of ESRD patients treated using this modality. ${ }^{4,5} \mathrm{HD}$ drastically and negatively affects the quality of life and life expectancy, with both physical and psychological effects. ${ }^{6,7}$ The experience of multiple losses, including kidney function, the global influence on family roles, work competence, sexual function, time, and mobility, significantly and negatively impact the lives of sufferers. ${ }^{6-9}$ Further stressors, including medication effects ${ }^{9}$ dietary constraints, fear of death, and dependency upon treatment ${ }^{10-12}$ may affect quality of life and exacerbate feelings of a loss of control. Depression is widely believed to be the 
most common psychopathological condition among patients with ESRD. ${ }^{9,13-15}$

While prevalent, depression is still often unrecognized, ${ }^{16}$ reflecting a lack of routine psychological evaluation among this patient population. ${ }^{17}$ The consequences of unidentified depression management among dialysis patients may be significant. Co-morbid depressive illnesses amplify the impact of chronic illnesses and increase functional disability and the use of health care services. ${ }^{18}$ Nephrologists are well-aware that the most common psychopathological conditions occurring in patients with HD are depression and depressive symptoms. ${ }^{9,13-15,19-21}$

Recent studies that employed Beck's Depression Inventory (BDI) have reported remarkably similar findings. ${ }^{2,21,22}$ The BDI is one of the most commonly used screening tools for depression in the dialysis population and is a self-rating tool that measures cognitive-affective symptoms and attitudes, impaired performance, and somatic symptoms. Furthermore, Wuerth et $\mathrm{al}^{23}$ observed that when these patients were subsequently evaluated by a trained psychiatric interviewer for the presence of clinical depression, $85 \%$ of dialysis patients with BDI scores of 11 or greater met the Diagnostic and Statistical Manual of Mental Disorders (DSM-IV) criteria for the diagnosis of major depression and had scores of 17 or more on the 21-item Hamilton Depression Scale. ${ }^{23}$

The BDI is a commonly employed tool for identifying depression, has been extensively validated in various studies, ${ }^{24-26}$ and has been compared with the Hamilton Depression Rating Scale and the Montgomery-Asburg Depression Rating Scale, which are both expert-administered scales. ${ }^{27-32}$ In identifying depression, the BDI has been reported to exhibit discriminative validity. ${ }^{26}$ Our results suggest that there is fair to good interpreter agreement in terms of presence of a depressed mood between patients and controls; agreement was highest among patients with the most intact cognition.

Even after years of study, researchers estimate that the prevalence of depression among dialysis patients varies widely from 5\%-50\%..$^{19,33-36}$ These differences have been attributed to varying criteria, methodology, and screening tools used to diagnose depression. ${ }^{1,3}$ The best estimates of prevalence are between $20 \%$ and $30 \%,{ }^{17,6}$ but the reported variations vary significantly due in part to the type of assessment tool employed. ${ }^{35,37}$

The prevalence of major depression in the general population is approximately $1.1 \%-15 \%$ for men and $1.8 \%-23 \%$ for women. ${ }^{6,38}$ However, the prevalence of major depression among ESRD patients is approximately 20\%-30\%, and it may be as high as $47 \% .^{13,14,38,39}$ Some studies have indicated that moderate depressive syndromes are common in approximately $25 \%$ of ESRD patients, and that major depression is common in 5\%-22\% of ESRD patients. ${ }^{40,41}$

As described above, the prevalence of depression in patients with HD varies according to the test being used. It has been found to be $50 \%$ in patients with HD using screening tools such as BDI, 10\% using the Multiple Affect Adjective Check List, but in only 5\% when a diagnosis was made by psychiatric interview using the American Psychiatric Association standards published in the DSM. Postulated explanations for these wide-ranging estimates vary among medical professionals; for example, in a British study, a primary nurse diagnosed depression in $41.9 \%$ of patients, but the nephrology team diagnosed depression in only $24.2 \%$. According to BDI scores, the rate was $38.7 \%{ }^{37}$

Additional reasons such as different cultural values influence estimated prevalence data of depression; for example, the Dialysis Outcomes and Practice Patterns Study data show that physician-diagnosed depression rates ranged from $2.0 \%$ in Japan to $21.7 \%$ in the United States, but the rate of self-reported depression in these countries using the Center for Epidemiological Studies Depression Screening Index (CES-D) was similar and much higher: $40.0 \%$ in Japan and $39.2 \%$ in the US. ${ }^{19}$ Additionally, timing of screen depression screening is important. Experts have cautioned that the first 3-6 months of dialysis is an unstable and critical period, and that levels of depression may change over time. ${ }^{17}$ Indeed, screening during the first 6 months of a patient's dialysis treatment is complicated because it is a very traumatic time. There is powerful, pervasive grief, and symptoms of uremia that must to be considered. Patients should be reassessed every 3-4 months to determine whether changes have occurred.

The etiology of dialysis-related depression is multifactorial and is related to biological, psychological, and social mechanisms. Biological mechanisms include increased cytokine levels ${ }^{6}$ and possible genetic predisposition. ${ }^{42,43}$ Psychological and social factors include feelings of hopelessness, perceptions of loss and lack of control, job loss, and altered family and social relationships. ${ }^{46}$ Another trigger of depression among HD patients is fatigue, which is a subjective symptom characterized by tiredness, weakness, and lack of energy. Approximately $60 \%-97 \%$ of patients on HD experience some fatigue, which is negatively correlated with quality of life..$^{44,45}$

\section{Relationship between depression and cortisol}

Depression is a very heterogeneous disorder with many different etiologies. By understanding the underlying 
causes of depression, progress towards effective treatment can be made.

Studies have demonstrated a connection between cortisol levels and depression. However, the results of many studies continue to both contradict each other as well as add to the current understanding.

Cortisol, a glucocorticoid hormone, is essential to human brain functioning. Produced by the adrenal glands, cortisol helps cells to create energy. When a body experiences stress, whether physical, mental, or emotional, cortisol production by the adrenals is upregulated in order to protect the body. This increase in cortisol is part of what is known as the "fight-or-flight" response, which occurs when a body prepares itself to combat a stressful event. This response is critical to an individual's ability to act quickly in emergency situations.

Rather than an "on-off" causation effect, studies continue to allude to a broad middle ground of impact between cortisol levels and depression. Balanced hormone levels, particularly regarding quantity and timing, appear to be important for mental health. Theories regarding the connection between cortisol and depression continue to be proposed.

In a study of 1588 participants, Vreeburg et $\mathrm{al}^{46}$ showed a possible increased biological propensity for depression when higher cortisol awakening responses were observed. This large cohort study showed significant, although modest, associations between major depressive disorder (MDD) and specific hypothalamic-pituitary-adrenal (HPA) axis indicators. Because a higher cortisol awakening response was observed among both subjects with current MDD and subjects with remitted MDD, an increased biological vulnerability for depression was indicated. ${ }^{46}$

It is known that in normal people, cortisol level in the bloodstream peaks in the morning, and then decreases as the day progresses. In depressed people, however, cortisol peaks earlier in the morning and does not level off or decrease in the afternoon or evening. Although the exact mechanism that causes depression is unknown, clinical studies suggest that chronically elevated cortisol may induce clinical depression by affecting central serotonergic neurotransmission. ${ }^{47,48}$

Because patients with major depression exhibit decreased brain serotonin (5-hydroxytryptamine [5-HT]) function and elevated cortisol secretion, it is generally accepted that changes in serotonin and cortisol are related to depression. A more recent study suggested that elevated cortisol levels, likely caused by stressful life events, may lower brain 5-HT function, leading to a depressive state. ${ }^{49}$ This study ties abnormalities in cortisol secretion and 5-HT function into a causal chain in which cortisol is the key biological mediator through which life stress lowers brain 5-HT function, causing depression in vulnerable individuals. ${ }^{49}$

Du's research team ${ }^{50}$ concluded not only that cortisol is necessary for coping, but also that chronically elevated glucocorticoids, as can occur during ongoing stressful situations, work against the body at a cellular level. Their article suggests that this chronic stress-related cell damage may cause physical and mental illness such as depression. ${ }^{50}$

Additionally, growing evidence suggests that specific types of chronic difficulty, such as caring for a relative with dementia, can be associated with increased cortisol secretion, ${ }^{51}$ indicating that life events and difficulties can result in cortisol hypersecretion, but that this does not necessarily lead to development of a depressive disorder.

However, some animal studies suggest that elevated cortisol levels may enhance 5-HT neurotransmission by decreasing the sensitivity of inhibitory $5-\mathrm{HT}_{1 \mathrm{~A}}$ cell body autoreceptors. ${ }^{52}$ From this viewpoint, elevated cortisol levels may promote resilience to environmental adversity. Some subjects may respond to 'stress levels' of cortisol by developing depression, but in these cases cortisol hypersecretion must interact with other predisposing factors. In most moderately depressed patients in primary care, increased cortisol secretion may not be an important pathophysiological factor. ${ }^{52}$

In contrast, other studies have shown that mice injected with cortisol developed symptoms of depression. They suggest that high cortisol levels may decrease serotonin function, leading to depression. ${ }^{53,54}$

Mackin and Young ${ }^{55}$ reported that abnormal cortisol levels were first observed in depressed patients in the late 1950s. Connections between endocrine (hormonal system) function and mental health have been explored for decades. As the understanding of the endocrine system has advanced with neurocognitive research, causes and potential treatments for mental illness have become increasingly clear. ${ }^{55}$

Quality of life domains include physical, psychological, and social functioning and general satisfaction with life. ${ }^{56}$ This article primarily measures the frequency of MDD and its predictive risk factors among the Arab population, as well as the issues surrounding screening, methodology, and the underlying causes and consequences of depression in the HD population.

\section{Methods}

A cross-sectional study was performed from October 1, 2010 to November 30, 2011 and included 71 patients (37 females) and 26 control subjects. Patients were included in the study 
if they had ESRD, were on maintenance HD for at least 3 months, and were 18 years of age or older. The control group included 26 control volunteers. The control group included HD patients' relatives, who were employed as caregivers at the same time by their HD relatives, with no history of depressive episodes. The control group was age-matched to HD patients, who were able to communicate easily and who agreed to answer questionnaires. Exclusion criteria included inability to participate in the psychiatric interview, acute renal failure, and delirium, patients with general mood disorders or complaints of cardiovascular disease, severe electrolyte disturbances, anemia, sepsis, and hormonal disturbances. Additionally, patients who were diagnosed with psychiatric disorders other than MDD based on psychiatric interviewing were excluded. All patients fulfilling the inclusion criteria who regularly visited our department for HD were included. A single psychiatric physician interviewed all study subjects. Subjects were screened for mood, anxiety, and somatoform disorders based on the DSM-IV diagnostic criteria. All participants underwent a series of interviews; demographic data and comprehensive clinical records were collected at study entry.

The study included two essential target goals. The first objective was to identify and measure the prevalence of major depressive disorder and its risk factors at the Nephrology Unit, EMMS hospital of Nazareth, Israel, after approval by the ethical committee of the hospital and obtaining signed consent from all participants. The second goal was to explore underlying etiologies and relationships between depression and hormonal disorders such as the glucocorticoid hormone (cortisol).

The Hamilton Depression Scale was administrated, and a score of 18 or greater on Hamilton Depression Scale accompanied by the presence of significant neurovegetative changes were evaluated to confirm the diagnosis. We also used the BDI, a self-administration evaluation including 21 questions, which is known to be reliable and valid, as the main evaluation method in our study. A BDI score of 11 or greater indicates the presence of at least a moderate level of depression symptoms. Patients with a Hamilton score of $>18$ and/or BDI score of $>11$ underwent a structured clinical interview by a psychiatrist based on DSM-IV criteria. Diagnosis was made using the DSM-IV to correlate psychological variables with clinical, hematological, and biochemical parameters. Independent variables included depression, socioeconomic situation, including income, education level, cortisol levels and C-reactive protein, albumin, and hospitalization rates. Dependent variables included HD patients, age, sex, and those who were unmarried and young females (Table 1).

MDD is defined by the DSM-IV, as the loss of pleasure or interest for at least 2 weeks, accompanied by five or more psychological, somatic, and behavioral symptoms. Several symptoms including anhedonia and feelings of sadness, emotional inhibitions, lack of energy, sleep disturbances, loss of concentration, intense guilt, and thoughts of suicide or death are associated with major depression. Thoughts of suicide and death are symptoms that are of obvious concern.

Table I Demographic and other different variables

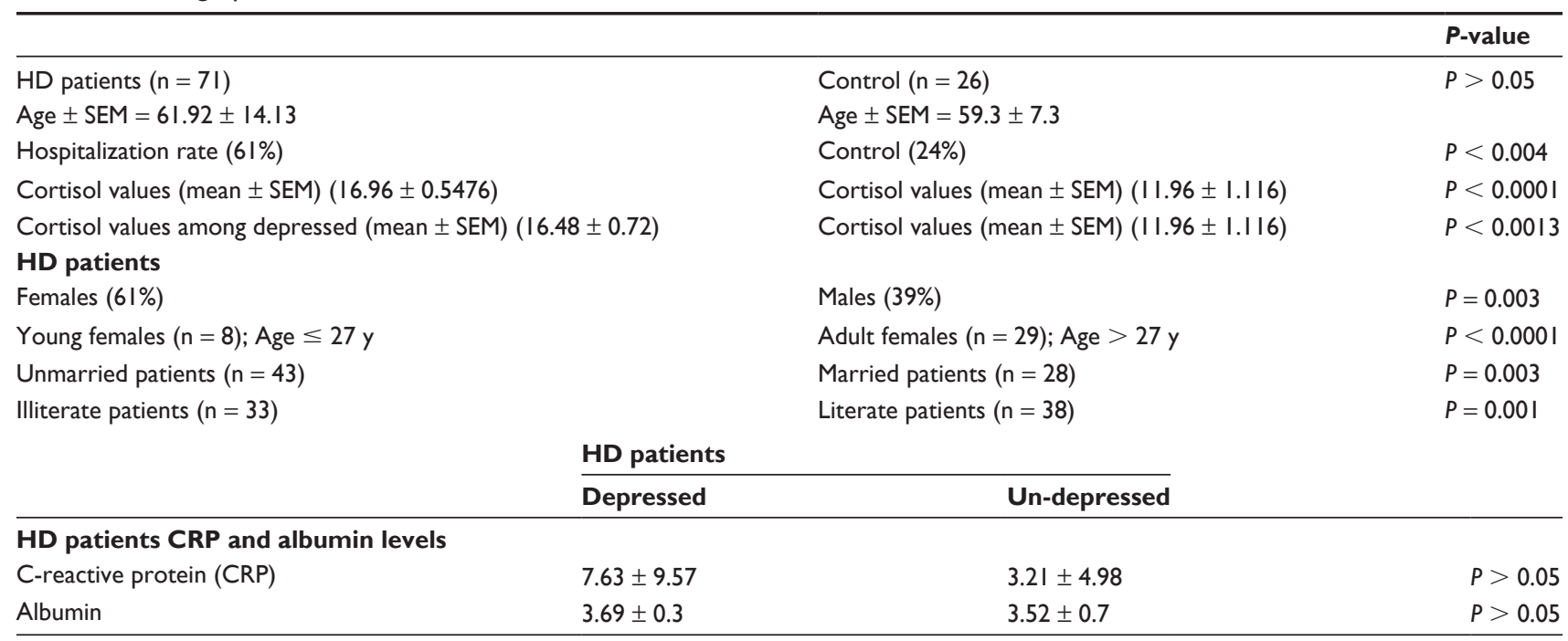

Notes: Statistical analysis which was performed on demographic and clinical parameters of the different tested groups. No significant difference was found in the average age of control group vs patient group. Neither CRP nor albumin levels were found to be statistically significant between subgroups of Depressed versus Un-depressed patients. Statistically significant effects were observed when we compared cortisol values among HD patients vs control; cortisol values among depressed patients vs control; Females vs males; young females vs adult females; unmarried patients vs married patients and illiterate vs literate patients.

Abbreviations: HD, hemodialysis; SEM, standard error of mean. 
Blood samples were drawn for routine hematological and biochemical parameters and viral markers (antihepatitis $\mathrm{C}$ and HbsAg). Norepinephrine was assayed using high-pressure liquid chromatography with electrochemical detection. Intra- and interassay coefficients of variability were $11.6 \%$ and $7.7 \%$. A blood exam for plasma cortisol levels was conducted early in the morning and in the afternoon. Cortisol was tested by using a radioimmunoassay. Intra- and interassay coefficients of variation were $6 \%$ and $10 \%$.

Statistical analysis carried out using one-way analyses of variance followed by Tukey's post-hoc multiple comparison tests were used. Finally, in order to characterize the relationship between depression scoring measures and the startle response, Pearson correlation was calculated.

\section{Results}

The mean ages of the chronically depressed HD patients and control subjects were $61.92 \pm 14.13$ and $59.3 \pm 7.3$ years $(P>0.05)$, respectively (Table 1$)$. The prevalence of severe depression among our HD patients, calculated using BDI, was $43.7 \%$. The frequency of depression was two-fold higher among young female patients $(n=8$, age $\leq 27$ years $)$ undergoing HD for at least 12 months compared to adulthood female HD patients $(\mathrm{n}=29$, age $>27$ years, $P<0.0001)$. Depression was twofold higher among unmarried patients $(\mathrm{n}=43)$ versus married patients $(\mathrm{n}=28, P=0.003)$, and threefold higher among illiterate patients $(\mathrm{n}=33)$ versus literate patients $(\mathrm{n}=38, P=0.001)$. Depression was also higher among females than among males ( $61 \%$ vs $39 \%, P=0.003)$. The standard of living and socioeconomic status of our patients were examined by a social worker, who concluded that nearly all patients required financial support for their disability after losing employment. A covariant connection was observed between joblessness $(n=66: 93 \%)$ and depression. When socioeconomic status deteriorated, the tendency for depression increased $(P<0.001)$. Hospitalization rates were higher among depressed HD patients compared to controls ( $61 \%$ vs $24 \%, P<0.004$, respectively) (Table 1 ). Comparing cortisol values between (HD) and control subjects, the levels were statistically significant for HD patients $(P<0.0001,95 \%$ CI: $2.416-6.825$; mean cortisol \pm SEM of patients; $n=71$, $16.96 \pm 0.5476$; for control subjects, $n=26,11.96 \pm 1.116$ ) (Figure 1). Comparing cortisol values between depressed HD patients and control subjects, the values were also statistically significant $(P=0.0013,95 \%$ CI: $1.868-7.184$; mean \pm SEM of depressed patients, $16.48 \pm 0.72$; for control subjects, $11.96 \pm 1.116$ ) (Figure 2). Comparing cortisol levels among depressed HD patients and nondepressed HD patients, the

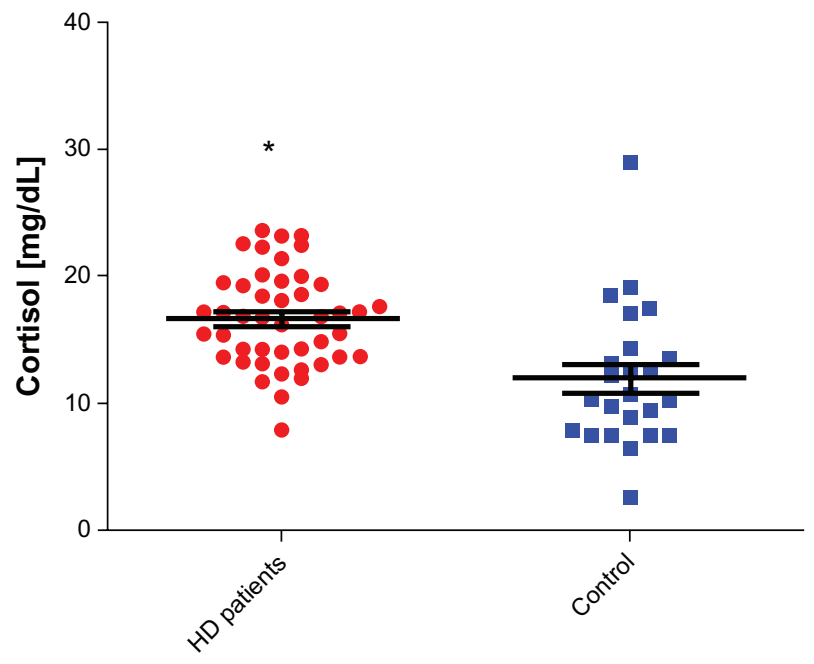

Figure I Cortisol levels among hemodialysis (HD) patients vs control. Note: Asterisk indicates the difference between the levels of cortisol among HD patients versus control was statistically significant in favor of HD patients.

results were statistically insignificant (Figure 3). Additionally, the levels of norepinephrine between depressed patients and control subjects were insignificant. C-reactive protein, albumin, hemoglobin, calcium, and phosphor values were compared between depressed HD patients and nondepressed HD patients, and the results were statistically insignificant $(P>0.05)$ (Table 1).

Despite strong familial ties present among Arabic traditions, this factor failed to decrease the depression among our patients.

\section{Discussion}

Depression has been identified as a complicating comorbid diagnosis in a variety of medical conditions, including ESRD.

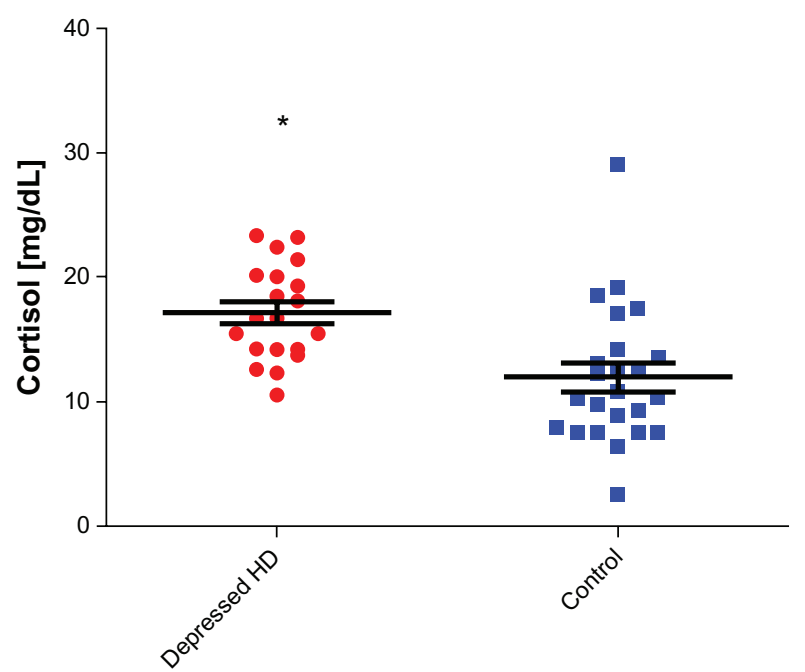

Figure 2 Cortisol levels among depressed hemodialysis (HD) patients vs controls. 


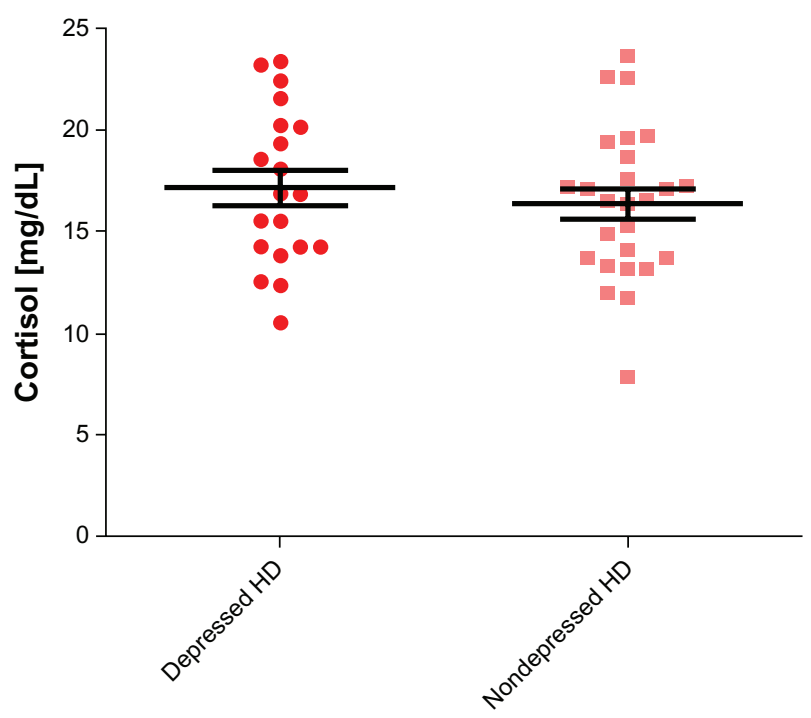

Figure 3 Cortisol levels among depressed hemodialysis (HD) vs nondepressed hemodialysis patients.

Depression is a common psychopathological inconvenience experienced by patients undergoing dialysis which can affect quality of life, morbidity, and mortality of this population, yet its detection and management are typically not documented as part of the routine care of this population. Nevertheless, increasing awareness of psychiatric problems for both patients and physicians, which are often overlooked by patients, their families, and physicians, may increase the early diagnosis and treatment of depressive disorders and may be important for successful care and adherence outcomes. Additionally, awareness of psychiatric problems helps to facilitate psychosocial adaptation of patients, reduce treatment-related costs by increasing treatment success, and decreasing hospitalizations.

In our study, patient and control groups, which had similar age and sex distributions, were compared by evaluating social, economic, and psychological factors. The high unmarried and illiteracy rates observed in our patient group can be coupled with the detrimental effects of depression. The frequency of depression was two-fold higher among unmarried patients compared to married patients, and three-fold more frequent among illiterate patients compared to literate patients. These findings agree with those of previous studies that also reported that a lack of social and familial support increases mortality. ${ }^{57,58}$ Indeed, being married and having a high-quality marriage are associated with lower depression scores compared to being single or widowed..$^{59,60}$

In agreement with several previous studies, higher depression scores are associated with low educational level. ${ }^{61,62}$
The standard of living and the socioeconomic status of nearly all our patients were low and sometimes intolerable, and nearly all required financial supports for their disability after losing employment. The reason for the high rates of unemployment may be due to HD treatment 3 to 4 hours three times per week, which is generally unacceptable to most employers. The patients also had more disease-related disability and more limited work opportunities due to their low-educational levels. This fact fits the covariance with depression, and has a negative impact on patient illness. According to previous studies, as socioeconomic status deteriorates, depression generally increases.$^{63}$

We also observed a high rate of hospitalization among our depressed HD patients compared to control subjects. This is related to the negative effect of depression in this group, and previous studies have extensively discussed this issue. ${ }^{63-65}$

Our results also showed a relatively high prevalence of severe depression in nearly half $(43.7 \%)$ of HD patients compared to control group subjects (15\%). Similar results were reported by Watnick et al. ${ }^{33}$ Additionally, depression frequency was high among females $(61 \%)$ compared with males (39\%); this difference may due to the sensitivity of females to stressors and their greater concern about the future. A female's obligations to adopt several social roles, such as a mother, wife, housewife, and business woman, may also contribute to this increased stress. In previous studies, both depression and anxiety were reported to be more frequent in females than in males. ${ }^{66,67}$

To be young, female, unemployed, on dialysis for less than 2 years, and living alone are suggested as risk factors for depression as reported by Craven et al. ${ }^{68}$

Another interesting finding of our study was the high level of serum cortisol among HP patients compared to that in control subjects $(P<0.0001)$, and the high cortisol levels among depressed HD patients versus control subjects $(P=0.0013)$. It is well-known that in normal physiology, the end product of the HPA axis is the glucocorticoid cortisol, secreted from the adrenal gland upon stimulation of adrenocorticotropin (ACTH) from the pituitary gland. ACTH is in turn secreted in response to corticotropin-releasing hormone and vasopressin from the hypothalamus. Cortisol exerts negative feedback control on both corticotropin-releasing hormone and vasopressin in the hypothalamus, and ACTH in the pituitary. In normal individuals, cortisol is secreted in a circadian rhythm; it is the loss of this circadian rhythm, together with loss of the normal feedback mechanism of the HPA axis, which results in chronic exposure to excessive circulating cortisol levels. 
Cortisol is widely known to be a stress hormone produced by the human body's adrenal glands. When the body is under stress, the adrenal gland increases secretion of cortisol. In the short-term, this hormone can aid in survival, for example by mobilizing energy reserves. Long-term elevation of cortisol, however, can have detrimental effects. The timing of cortisol release can cause problems in people who are depressed. It is known that normal plasma cortisol levels fluctuate in a diurnal pattern, with maximal levels of up to $(5-25 \mu \mathrm{g} / \mathrm{mL})$ at $8: 00$ am and $4: 00 \mathrm{pm}$, and lowest during the night $(2.9-13 \mu \mathrm{g} / \mathrm{mL})$. A depressed individual may have a consistent level of cortisol or high amounts in the middle of the night. In depressed people, however, cortisol peaks earlier in the morning and does not level off or decrease in the afternoon or evening. Although the exact mechanism that causes depression is unclear, clinical studies suggest that chronically elevated cortisol may induce clinical depression by affecting central serotonergic neurotransmission. ${ }^{48,69}$ In fact, it has long been observed that a significant proportion of depressed patients have elevated plasma cortisol levels, ${ }^{69}$ possibly due to abnormalities in negative feedback at multiple levels in the HPA axis. ${ }^{70}$ It is likely that during critical illness, under chronic stress or depression, glucocorticoid secretion and cortisol levels markedly increase. ${ }^{71-73}$

High circulating cortisol levels may also be related to the kidneys. Indeed, the kidney plays a significant role in degrading many peptides and hormones. ${ }^{74,75}$ Severe chronic renal failure is associated with a prolonged $\mathrm{Tl} / 2$ of serum cortisol, which can be returned to normal or near normal by dialysis. ${ }^{76}$ However, compared to basal cortisol levels of normal subjects, HD patients were relatively hypercortisolemic. This finding was also confirmed by previous studies. ${ }^{77-79}$ Since a small percentage of cortisol metabolites can be detected by measuring plasma cortisol, our findings could, in part, be due to high plasma cortisol metabolites which can accumulate in patients with ESRD. ${ }^{80}$

Other previous findings in HD patients suggested a defect in the hypothalamic reactivity for ACTH secretion, as well as a defect in adrenal cortisol secretion possibly due to a biologically inactive form of ACTH. ${ }^{78-81}$ Indeed, depressed patients have increased tissue and central nervous system exposure to free, physiologically active glucorticoides. The appearance of severe depressive symptoms which manifests a diurnal rhythm may be due in part by excessive central nervous system exposure to glucocorticoids. ${ }^{82}$ Whatever the reasons for the increased level of cortisol among our HD patients, measuring serum cortisol concentrations in HD patients with depression may help in the detection of the disease in its early stages and contribute to a quick diagnosis to permit early therapeutic interventions. All of our depressed HD patients were treated using antidepressants. The systematic use of screening instruments for depressive symptoms may help medical personnel identify hemodialysis patients who require special care in order to improve their quality of life, reduce hospitalization, and increase survival.

In our study the BDI, was an easily administered questionnaire and is a useful screen for identifying potentially treatable clinical depression. Employment of BDI, the Hamilton Depression Scale, and the DSM-IV, have reported remarkable accuracy as screening and diagnostic tools for depression among patients with HD. Their specificity and accuracy were justified by the high prevalence of depression observed among HD patients.

Further investigation of the BDI in dialysis populations is required before conclusions can be drawn on the appropriateness of this methodology. We suggest that on-dialysis screening is viable, and that the BDI is a suitable screening tool. Because of the limited sample size of our study, we cannot exclude the possibility that the observed effects are due to confounding factors that could not be fully evaluated due to the limited power of our method (ie, differences between depressed and nondepressed subjects). Further studies examining the turnover activity of glucocorticoids such as cortisol are needed to confirm the effectiveness of glucocorticoids as models or markers for early diagnosis of depression among HP patients.

In summary, our data suggest that most patients undergoing HD are severely depressed and that screening and diagnosis of HD patients for depression is extremely important for successful therapy adherence. We have instituted a program in our dialysis units to screen patients every 6 months to identify those with BDI scores of 11 or greater, or/and with a Hamilton Depression score of 17 or more on the 21 Hamilton Depression Scale. All patients with BDI scores of 11 or greater, or/and with Hamilton Depression Scale of 17 or more are referred to a trained psychiatric interviewer. If the patient meets the DSM-IV criteria for depression, antidepressant medication is prescribed and the patient is carefully monitored. BDI is an easily administered questionnaire that is a useful screen for potentially treatable clinical depression. High level of glucocorticoid hormones such as cortisol turnover may be used as indices for early detection and diagnosis of depression among HD patients. 


\section{Disclosure}

The authors declare that they have no competing interests. $\mathrm{ZH}$ participated in writing part of the conclusion and study design, editing the manuscript, and helped to draft the manuscript. BB carried out the study design and participated in patient recruitment as well as editing of the manuscript and funding the project. AA participated in statistical analysis, study design, and data collection. SS participated in the study design, created tables and figures, and helped with statistical analysis. ZM participated in collection of data and lab analysis. HE participated in lab analysis and patient recruitment. JA was the lab director and participated in data analysis. FJ participated partially in the data collection and helped with study design. BA conceived of the study, and participated in its design and coordination; wrote nearly all of the manuscript (Introduction and Discussion) and helped to draft the manuscript.

\section{References}

1. Finkelstein FO, Finkelstein SH. Psychological adaptation and quality of life of the patient with end-stage renal disease. In: Brown E, Parfrey P, editors. Complications of Long Term Dialysis. Oxford University Press: Oxford; 1999:168-187.

2. Kimmel PL, Peterson RA, Weihs KL, et al. Multiple measurements of depression predict mortality in a longitudinal study of chronic hemodialysis patients. Kidney Int. 2000;57(5):2093-2098.

3. Kimmel PL. Psychosocial factors in adult end-stage renal disease patients treated with hemodialysis: correlates and outcomes. Am J Kid Dis. 2000;35(1):132-140.

4. Collins AJ, Kasiske B, Herzog C, et al. Excerpts from the United States Renal Data System 2006 Annual Data Report: Atlas of End-Stage Renal Disease in the United States. Am J Kidney Dis. 2007;49(Suppl 1):vi-vii: S1-S296.

5. Foley RN, Collins AJ. End-stage renal disease in the United States: an update from the United States Renal Data System. J Am Soc Nephrol. 2007;18(10):2644-2648.

6. Kimmel PL. Psychosocial factors in dialysis patients. Kidney Int. 2001;59(4):1599-1613.

7. Reiss D. Patient, family, and staff responses to end-stage renal disease. Am J Kidney Dis. 1990;15(3):194-200.

8. Chilcot J, Wellsted D, Da Silva-Gane M, Farrington K. Depression on dialysis. Nephron Clin Pract. 2008;108(4):c256-c264.

9. Kimmel PL. Depression in patients with chronic renal disease: what we know and what we need to know. J Psychosom Res. 2002; 53(4):951-956.

10. De-Nour AK, Shaltiel J, Czaczkes JW. Emotional reactions of patients on chronic hemodialysis. Psychosom Med. 1968;30(5):521-533.

11. O'Brien ME. Compliance behavior and long-term maintenance dialysis. Am J Kidney Dis. 1990;15(3):209-214.

12. De-Nour AK. Social adjustment of chronic dialysis patients. Am J Psychiatry. 1982;139(1):97-100.

13. Kimmel PL, Weihs K, Peterson RA. Survival in hemodialysis patients: the role of depression. J Am Soc Nephrol. 1993(1);4:12-27.

14. Finkelstein FO, Finkelstein SH. Depression in chronic dialysis patients: assessment and treatment. Nephrol Dial Transplant. 2000; 15(12):1911-1913.

15. Levenson JL, Glocheski S. Psychological factors affecting endstage renal disease: a review. Psychosomatics. 1991;32(4): $382-389$.
16. Watnick S, Wang PL, Demadura T, Ganzini L. Validation of 2 depression screening tools in dialysis patients. Am J Kidney Dis. 2005;46(5):919-924.

17. Killingworth A, van den Akker O. The quality of life of renal dialysis patients: trying to find the missing measurement. Int J Nurs Stud. 1996;33(1):107-120.

18. Stein MB, Cox BJ, Afifi TO, Belik SL, Sareen J. Does co-morbid depressive illness magnify the impact of chronic physical illness? A population-based perspective. Psychol Med. 2006;36(5):587-596.

19. Lopes AA, Albert JM, Young EW, et al. Screening for depression in hemodialysis patients: associations with diagnosis, treatment, and outcomes in the DOPPS. Kidney Int. 2004;66(6):2047-2053.

20. Cilan H, Oguzhan N, Unal A, et al. Relationship between depression and proinflammatory cytokine levels in hemodialysis patients. Ren Fail. 2012;34(3):275-278.

21. Steele TE, Baltimore D, Finkelstein SH, Juergensen P, Kliger AS, Finkelstein FO. Quality of life in peritoneal dialysis patients. J Nerv Ment Dis. 1996;184(6):368-374.

22. Beck AT, Steer RA, Brown GK. Beck Depression Inventory. 2nd ed. San Antonio: The Psychological Corporation; 1996.

23. WuerthDB, Finkelstein SH, Schwetz O, Carey H, KligerAS, Finkelstein FO. Patients' descriptions of specific factors leading to modality selection of chronic peritoneal dialysis or hemodialysis. Perit Dial Int. 2002;22(2):184-190.

24. Lambert MJ, Hatch DR, Kingston MD, Edwards BC. Zung, Beck, and Hamilton rating scales as measures of treatment outcome: a meta-analytic comparison. J Consult Clin Psychol. 1986;54(1):54-59.

25. Snaith RP, Taylor CM. Rating scales for depression and anxiety: a current perspective. Br J Clin Pharmacol. 1985;19(Suppl 1):S17-S20.

26. Plumb MM, Holland J. Comparative studies of psychological function in patients with advanced cancer-I. self-reported depressive symptoms. Psychosom Med. 1977;39(4):264-276.

27. De Souza J, Jones LA, Rickards H. Validation of self-report depression rating scales in Huntington's disease. Mov Disord. 2010;25(1): 91-96.

28. Cukor D, Peterson RA, Cohen SD, Kimmel PL. Depression in endstage renal disease hemodialysis patients. Nat Clin Pract Nephrol. 2006;2(12):678-687.

29. Craven JL, Rodin GM, Littlefield C. The Beck Depression Inventory as a screening device for major depression in renal dialysis patients. Int J Psychiatry Med. 1988;18(4):365-374.

30. Cohen SD, Norris L, Acquaviva K, Peterson RA, Kimmel PL. Screening, diagnosis, and treatment of depression in patients with end-stage renal disease. Clin J Am Soc Nephrol. 2007;2(6):1332-1342.

31. Gençöz F, Tülin Gençöz T, Soykan A. Psychometric properties of the Hamilton Depression Rating Scale and other physician-rated psychiatric scales for the assessment of depression in ESRD patients undergoing hemodialysis in Turkey. Psychol Health Med. 2007; 12(4):450-459.

32. Zimmerman M, Chelminski I, Posternak M. A review of studies of the Hamilton depression rating scale in healthy controls: implications for the definition of remission in treatment studies of depression. J Nerv Ment Dis. 2004;192(9):595-601.

33. Watnick S, Kirwin P, Mahnensmith R, Concato J. The prevalence and treatment of depression among patients starting dialysis. Am J Kidney Dis. 2003;41(1):105-110.

34. Weisbord SD, Fried LF, Unruh ML, et al. Associations of race with depression and symptoms in patients on maintenance haemodialysis. Nephrol Dial Transplant. 2007;22(1):203-208.

35. Kimmel PL, Peterson RA. Depression in end-stage renal disease patients treated with hemodialysis: tools, correlates, outcomes, and needs. Semin Dial. 2005;18(2):91-97.

36. Wilson B, Spittal J, Heidenheim P, et al. Screening for depression in chronic hemodialysis patients: comparison of the Beck Depression Inventory, primary nurse, and nephrology team. Hemodial Int. 2006; 10(1):35-41. 
37. Smith MD, Hong BA, Robson AM. Diagnosis of depression in patients with end-stage renal disease. Comparative analysis. Am J Med. 1985;79(2):160-166.

38. Drayer RA, Piraino B, Reynolds CF 3rd, et al. Characteristics of depression in hemodialysis patients: symptoms, quality of life, and mortality risk. Gen Hosp Psychiatry. 2006;28(4):306-312.

39. Kim JA, Lee YK, Huh WS, et al. Analysis of depression in continuous ambulatory peritoneal dialysis patients. J Korean Med Sci. 2002;17(6):790-794.

40. Herrmann-Lingen C, Klemme H, Meyer T. Depressed mood, physician-rated prognosis, and comorbidity as independent predictors of 1-year mortality in consecutive medical inpatients. $J$ Psychosom Res. 2001;50(6):295-301.

41. Cohen LM, Dobscha SK, Hails KC, Pekow PS, Chochinov HM. Depression and suicidal ideation in patients who discontinue the life-support treatment of dialysis. Psychosom Med. 2002;64(6):889-896.

42. Israel M. Depression in dialysis patients: a review of psychological factors. Can J Psychiatry. 1986;31(5):445-451.

43. Smogorzewski M, NiZ, Massry SG. Function and metabolism of brain synaptosomes in chronic renal failure. Artif Organs. 1995;19(8):795-800.

44. Jhamb M, Weisbord SD, Steel JL, Unruh M. Fatigue in patients receiving maintenance dialysis: a review of definitions, measures, and contributing factors. Am J Kidney Dis. 2008;52(2):353-365.

45. Lee BO, Lin CC, Chaboyer W, Chiang CL, Hung CC. The fatigue experience of haemodialysis patients in Taiwan. J Clin Nurs. 2007; 16(2):407-413.

46. Vreeburg SA, Hoogendijk WJG, van Pelt J, et al. Major depressive disorder and hypothalamic-pituitary-adrenal axis activity. Arch Gen Psychiatry. 2009;66(6):617-626.

47. Heinz A, Jones DW, Bissette G, et al. Relationship between cortisol and serotonin metabolites and transporters in alcoholism (correction of alcolholism). Pharmacopsychiatry. 2002;35(4):127-134.

48. Tafet GE, Idoyaga-vargas VP, Denise P, et al. Correlation between cortisol level and serotonin uptake in patients with chronic stress and depression. Cognit Affect Behav Neurosci. 2001;1(4):388-393.

49. Dinan TG. Glucocorticoids and the genesis of depressive illness. A psychobiological model. Br J Psychiatry. 1994;164(3):365-371.

50. Du J, Wang Y, Hunter R, et al. Dynamic regulation of mitochondrial functions by glucocorticoids. Proc Natl Acad Sci U S A. 2009;106(9):3543-3548.

51. Bauer ME, Vedhara K, Perks P, Wilcock GK, Lightman SL, Shanks N. Chronic stress in caregivers of dementia patients is associated with reduced lymphocyte sensitivity to glucocorticoids. J Neuroimmunol. 2000;103(1):84-92.

52. McAllister-Williams RH, Anderson RJ, Young AH. Corticosterone selectively attenuates 8 -OH-DPAT-mediated hypothermia in mice. Int J Neuropsychopharmacol. 2001;4(1):1-8.

53. Ardayfio P, Kim KS. Anxiogenic-like effect of chronic corticosterone in the light-dark emergence task in mice. Behav Neurosci. 2006;120(2): 249-256.

54. Cowen PJ. Cortisol, serotonin and depression: all stressed out? $\mathrm{Br} J$ Psychiatry. 2002;180:99-100.

55. Mackin P, Young AH. The role of cortisol and depression: exploring new opportunities for treatments. Psychiatr Times. 2004;21(6):92-95.

56. Tsay SL, Healstead M. Self-care, self-efficacy, depression, and quality of life among patients receiving hemodialysis in Taiwan. Int $J$ Nurs Stud. 2002;39(3):245-251.

57. Christensen AJ, Wiebe JS, Smith TW, Turner CW. Predictors of survival among hemodialysis patients: effect of perceived family support. Health Psychol. 1994;13(6):521-525.

58. Kimmel PL, Peterson RA, Weihs KL, et al. Psychosocial factors, behavioral compliance and survival in urban hemodialysis patients. Kidney Int. 1998;54(1):285.

59. Osler M, McGue M, Lund R, Christensen K. Marital status and twins' health and behavior: an analysis of middle-aged Danish twins. Psychosom Med. 2008;70(4):482-487.
60. Holt-Lunstad J, Birmingham W, Jones BQ. Is there something unique about marriage? The relative impact of marital status, relationship quality, and network social support on ambulatory blood pressure and mental health. Ann Behav Med. 2008;35(2):239-244.

61. Hamad R, Fernald LC, Karlan DS, Zinman J. Social and economic correlates of depressive symptoms and perceived stress in South African adults. J Epidemiol Community Health. 2008;62(6): 538-544.

62. Di Corrado D, Di Nuovo S, Iannetti E, et al. Quality of life in hemodialyis patients: the effect of educational status. Clin Ter. 2000; 151(4):235-239.

63. Murata C, Kondo K, Hirai H, Ichida Y, Ojima T. Association between depression and socio-economic status among community-dwelling elderly in Japan: the Aichi Gerontological Evaluation Study (AGES). Health Place. 2008;14(3):406-414.

64. Lopes AA, Bragg J, Young E, et al. Depression as a predictor of mortality and hospitalization among hemodialysis patients in the United States and Europe. Kidney Int. 2002;62(1):199-207.

65. Christensen AJ, Ehlers SL. Psychological factors in end-stage renal disease: an emerging context for behavioral medicine research. $J$ Consult Clin Psychol. 2002;70(3):712-724.

66. Toros F, Bilgin NG, Bugdayci R, Sasmaz T, Kurt O, Camdeviren H. Prevalence of depression as measured by the CBDI in a predominantly adolescent school population in Turkey. Eur Psychiatry. 2004;19(5):264-271.

67. Schmaus BJ, Laubmeier KK, Boquiren VM, Herzer M, Zakowski SG Gender and stress: differential psychophysiological reactivity to stress reexposure in the laboratory. Int J Psychophysiol. 2008; 69(2):101-106.

68. Craven JL, Rodin GM, Johnson L, Kennedy SH. The diagnosis of major depression in renal dialysis patients. Psychosom Med. 1987;49(5):482-492.

69. Gibbons JL. Cortisol secretion rate in depressive illness. Arch Gen Psychiatry. 1964;10:572-575.

70. Holsboer F, Lauer CJ, Schreiber W, Krieg JC. Altered hypothalamicpituitary-adrenocortical regulation in healthy subjects at high familial risk for affective disorders. Neuroendocrinology. 1995;62(4): 340-347.

71. Epel ES. Psychological and metabolic stress: a recipe for accelerated cellular aging? Hormones (Athens). 2009;8(1):7-22.

72. Sonikian M, Metaxaki P, Papavasileiou D, et al. Effects of interleukin-6 on depression risk in dialysis patients. Am J Nephrol. 2010;31(4):303-308.

73. Hamrahian AH, Oseni TS, Arafah BM. Measurements of serum free cortisol in critically ill patients. N Engl J Med. 2004;350(16):1629-1638.

74. Feldman HA, Singer I. Endocrinology and metabolism in uremia and dialysis: a clinical review. Medicine. 1974;54(5):345-376.

75. Emmanouel DS, Lindheimer MD, Katz AI. Pathogenesis of endocrine abnormalities in uremia. Endocr Rev. 1980;1(1):28-44.

76. Bacon GE, Kenny FM, Mardaugh HV, Richards C. Prolonged serum half-life of cortisol in renal failure. Johns Hopkins Med J. 1973; 132(2):127-131.

77. Siamopoulos KC, Eleftheriadis E, Pappas M, Sferopoulos G, Tsolas O. Ovine corticotropin-releasing hormone stimulation test in patients with chronic renal failure: pharmacokinetic properties, and plasma adrenocorticotropic hormone and serum cortisol responses. Horm Res. 1988;30(1):17-21.

78. McDonald WJ, Golper TA, Mass RD, et al. Adrenocorticotropin-cortisol axis abnormalities in hemodialysis patients. J Clin Endocr Metab. 1979;48(1):92-95.

79. Wallace EZ, Rosman P, Toshav N, Sacerdote A, Balthazar A. Pituitaryadrenocortical function in chronic renal failure: studies of episodic secretion of cortisol and dexamethasone suppressability. J Clin Endocr Metab. 1980;50(1):46-51.

80. EnglertE Jr, Brown H, Willardson DG, Wallach S, Simons EL. Metabolism of free and conjugated 17-hydroxycorticosteroids in subjects with uremia. J Clin Endocr Metab. 1958;18(1):36-48. 
81. Ramirez G, Gomez-Sanchez C, Meikle WA, Jubiz W. Evaluation of the hypothalamic hypophyseal adrenal axis in patients receiving long term hemodialysis. Arch Intern Med. 1982;142(8):1448-1452.
82. Bernard JC, George CC, Mendels J. Cerebrospinal fluid and plasma free cortisol concentrations in depression. Psychol Med. 1976; 6(2):235-244.

\section{Publish your work in this journal}

Neuropsychiatric Disease and Treatment is an international, peerreviewed journal of clinical therapeutics and pharmacology focusing on concise rapid reporting of clinical or pre-clinical studies on a range of neuropsychiatric and neurological disorders. This journal is indexed on PubMed Central, the 'PsycINFO' database and CAS.
The manuscript management system is completely online and includes a very quick and fair peer-review system, which is all easy to use. Visit http://www.dovepress.com/testimonials.php to read real quotes from published authors.

\footnotetext{
Submit your manuscript here: http://www.dovepress.com/neuropsychiatric-disease-and-treatment-journal
} 